\section{MONITORAMENTO DE FATORES DE RISCO PARA DOENÇAS CRÔNICAS NÃO TRANSMISSÍVEIS: UM ESTUDO DE BASE POPULACIONAL}

\author{
Monitoring risk factors for noncommunicable diseases: a \\ population-based study
Monitorización de factores de riesgo para enfermedades crónicas no transmisibles: un estudio de base poblacional

\section{RESUMO}

Objetivo: Monitorar a prevalência dos fatores de risco à ocorrência das doenças crônicas não transmissíveis em adultos de um município do norte do Paraná, Brasil. Métodos: Tratase de um estudo transversal de base populacional, realizado em 2013 e operacionalizado por meio de uma entrevista domiciliar com 453 adultos selecionados conforme as áreas de extensão demográficas do município. As variáveis estudadas foram àquelas relacionadas às condições sociodemográficas e aos principais fatores de risco associados às doenças crônicas como tabagismo, atividade física, ingestão de bebida alcoólica e estado nutricional. Realizou-se análise bivariada das variáveis em cada nível de determinação utilizando P-valor $<0,05$. Resultados: Houve associação entre os fatores de risco para as doenças crônicas não transmissíveis e as características sociodemográficas para sexo, escolaridade e situação conjugal $(\mathrm{p}<0,05)$. A proporção da inatividade física ocorreu nos indivíduos do sexo feminino, com baixa escolaridade e entre os viúvos (as). Quanto ao consumo abusivo de álcool, os indivíduos do sexo masculino, com baixa escolaridade e entre os solteiros (as) tiveram significância estatística. O cigarro foi prevalente entre os homens e os solteiros (as) e o excesso de peso entre os indivíduos do sexo masculino, baixa escolaridade e casados (as), com significância estatística para essas variáveis. Conclusão: As características sociodemográficas mostraram associação entre os principais fatores de risco para as DCNT corroborando com a hipótese de que tais doenças também podem ter sua origem nos determinantes sociais.

Descritores: Doença Crônica; Fatores de Risco; Epidemiologia; Prevenção de Doenças.

\section{ABSTRACT}

Objective: To monitor the prevalence of risk factors for the onset of noncommunicable diseases in adults from a municipality in Northern Parana, Brazil. Methods: This is a crosssectional population-based study conducted in 2013 through home interviews with 453 adults selected according to the demographic extension areas of the municipality. The variables studied were those related to sociodemographic conditions and main risk factors associated with chronic diseases such as smoking, physical activity, alcohol consumption and nutritional status. Bivariate analysis of variables was performed at each level of measurement using $P$-value $<0.05$. Results: There was an association between risk factors for noncommunicable diseases and sociodemographic characteristics such as gender, education and marital status $(p<0.05)$. The proportion of physical inactivity was found in women, individuals with low education and widowed people. Regarding the abusive consumption of alcohol, men, individuals with low education and single people presented statistical significance. Smoking was prevalent among men and single individuals and excessive weight was prevalent among men, individuals with low education and married people, with statistical significance for these variables. Conclusion: The sociodemographic characteristics showed an association between the main risk factors for NCDs, corroborating the hypothesis that these diseases may also have their origin in the social determinants.

Descriptors: Chronic Disease; Risk Factors; Epidemiology; Disease Prevention.
Artigo Original
1) Universidade Federal da Fronteira Sul UFFS - Chapecó (SC) - Brasil

2) Escola Paulista de Enfermagem - Universidade Federal de São Paulo UNIFESP - São Paulo (SP) - Brasil

3) Universidade Federal do Rio Grande do Sul - UFRGS - Porto Alegre (RS) - Brasil
Recebido em: 28/02/2016 Revisado em: 05/03/2016 Aceito em: 30/04/2016 


\section{RESUMEN}

Objetivo: Monitorear la prevalencia de los factores de riesgo para la ocurrencia de enfermedades crónicas no transmisibles (ECNT) en adultos de un municipio del norte de Paraná, Brasil. Métodos: Se trata de un estudio transversal de base poblacional realizado en 2013 a través de una entrevista en el domicilio de 453 adultos elegidos según las aéreas de extensión demográficas del municipio. Las variables estudiadas fueron aquellas relacionadas con las condiciones socio demográficas y los principales factores de riesgo asociados con las enfermedades crónicas como el tabaquismo, la actividad física, la ingesta de bebida alcohólica y el estado nutricional. Se realizó un análisis bivariado de las variables de cada nivel de determinación utilizando el $p<0,05$. Resultados: Hubo asociación entre los factores de riesgo para las enfermedades crónicas no transmisibles y las características socio demográficas para el sexo, la escolaridad y la situación conyugal $(p<0,05)$. La proporción de la inactividad física se dio en los individuos del sexo femenino, de baja escolaridad y entre los viudos (as). Sobre el consumo abusivo del alcohol los individuos del sexo masculino, de baja escolaridad y entre los solteros (as) tuvieron estadística significativa. El tabaco fue prevalente entre los hombres y los solteros (as) y el exceso de peso entre los individuos del sexo masculino, baja escolaridad y casados (as) con significancia estadística para esas variables. Conclusión: Las características socio demográficas señalaron la asociación entre los principales factores de riesgo para las ECNT apoyando la hipótesis de que tales enfermedades también pueden tener su origen en los determinantes sociales.

Descriptores: Enfermedad Crónica; Factores de Riesgo; Epidemiologia; Prevención de Enfermedades

\section{INTRODUÇÃO}

A transição demográfica vivenciada no Brasil reflete as mudanças ocorridas no paradigma das doenças recorrentes na população. Tais mudanças podem ser atribuídas às modificações sociais e econômicas que têm influenciado a maneira de viver, trabalhar e se alimentar da sociedade, o que cooperou para o aumento da prevalência das Doenças Crônicas Não Transmissíveis (DCNT) no país ${ }^{(1)}$.

As DCNT são definidas como um conjunto de doenças multifatoriais que se desenvolvem no decorrer da vida, de etiologias incertas, longos períodos de latência e diferentes fatores de risco $^{(2)}$. Entre as doenças mais frequentes deste grupo, encontram-se a diabetes, as neoplasias, as doenças cardiovasculares e as doenças respiratórias crônicas ${ }^{(3)}$. Atualmente, são consideradas um sério problema de saúde pública responsáveis por $63 \%$ das mortes no mundo ${ }^{(2)}$. Seguindo a tendência mundial, no Brasil, as DCNT compõem a principal causa de morte em adultos e idosos e são responsáveis pelo aumento dos gastos com serviços, medicamentos e internações, custeando para o sistema público R \$ 4,18 milhões por ano ${ }^{(4,5)}$.

Essas patologias ameaçam a saúde e o desenvolvimento humano, uma vez que acarretam impactos na qualidade de vida dos indivíduos elevando a possibilidade de uma morte prematura, bem como aumentando os custos econômicos adversos para as famílias, comunidades e sociedade em geral $^{(6,7)}$. Tais doenças são resultados de diversos fatores determinantes sociais e condicionantes, além de fatores de risco individuais como tabagismo, consumo nocivo de álcool, inatividade física e alimentação não saudável ${ }^{(8)}$.

A atual política de assistência à saúde no país tem priorizado o investimento e a reorganização dos serviços em frente do desenvolvimento e controle das DCNT por meio da elaboração de estratégias e no delineamento de diretrizes em vigilância, informação, avaliação, monitoramento, promoção da saúde e cuidado integral ${ }^{(2)}$. A partir de então, estudos vêm sendo desenvolvidos a fim de se avaliar os fatores de risco associados à ocorrência ou não dessas doenças demonstrando, mesmo que indiretamente, a qualidade de vida dessa população $0^{(3,9)}$.

O compartilhamento desses fatores, somado à urgência em deter o crescimento das DCNT, justifica a adoção de estratégias integradas e sustentáveis por meio da vigilância e monitoramento do perfil dessas doenças e de seus fatores determinantes e condicionantes, bem como na detecção de mudanças em suas tendências no tempo, no espaço geográfico e em grupos populacionais, contribuindo assim para o planejamento de ações na área da saúde ${ }^{(10,11)}$.

Dessa forma, conhecer os fatores de risco mais prevalentes em uma população possibilita ações de saúde voltadas à prevenção, com consequente diminuição da incidência, mortalidade e melhoria da qualidade de vida de uma população, o que justifica a realização da presente pesquisa. Além disso, a análise da relação entre os fatores de risco existentes no desenvolvimento das DCNT pode gerar informações importantes para fomentar e apoiar o desenvolvimento e o fortalecimento da vigilância integrada dessas doenças por meio do aprimoramento de instrumentos de monitoramento desses fatores. Nessa perspectiva, evidencia-se a necessidade de aprofundar a análise sobre as DCNT e seus fatores associados. Ao identificar tais fatores é possível a construção de melhorias nessa área e contribuir para profissionais da saúde insiram novas condutas e rotinas no atendimento dessa população visando ao desencadeamento de intervenções e ações articuladas que promovam e estimulem a adoção de comportamentos e de estilo de vida saudáveis. Apesar de ser um tema bastante explorado na literatura são necessárias investigações que apresentem resultados quanto aos fatores de risco associados 
à ocorrência das doenças crônicas não transmissíveis. O Ministério da Saúde (MS) fomenta e incentiva a realização de pesquisas sobre o controle integrado de DCNT e seus fatores de risco em consonância com a Política Nacional de Promoção da Saúde e destaca que ainda existem muitos desafios no sentido da qualificação das ações e das respostas aos portadores das $\mathrm{DCNT}^{(2)}$.

Assim, este estudo parte do pressuposto que as DCNT também podem ter sua origem nos determinantes sociais, como renda, escolaridade, condições de moradia já que o processo saúde-doença pode ser socialmente determinado $^{(12)}$. Diante do exposto, objetivou-se monitorar a prevalência dos fatores de risco à ocorrência das doenças crônicas não transmissíveis em adultos de um município do norte do Paraná, Brasil.

\section{MÉTODOS}

Trata-se de um estudo transversal de base populacional realizado em 2013 por meio de entrevista domiciliar no município de Maringá, polo do norte do estado do Paraná, Brasil, com uma população estimada para o corrente ano de 397.437 habitantes $^{(13)}$. Com o objetivo de assegurar a representatividade do evento, foi utilizado cálculo amostral. A amostra consistiu na utilização das informações do censo demográfico referente à população adulta (idade $\geq 18$ anos) no ano anterior à coleta de dados (censo 2012), equivalente a 346.744 pessoas $^{(14)}$. Além disso, a seleção da amostra teve como unidade de referência as Áreas de Extensão Demográficas (AED) do município totalizando 21 áreas ${ }^{(13)}$.

Assim, o tamanho da amostra calculado no programa estatístico Epi-Info versão 3.5.1 incluiu a proporção da população adulta do município, com um erro de estimativa de $5 \%$ e confiabilidade de $95 \%$, acrescidos de $20 \%$ para possíveis perdas. Mediante a estratificação proporcional pelo número de indivíduos de cada $\mathrm{AED}$, a amostra final resultou em 453 participantes.

A seleção para a representatividade amostral se deu por demanda espontânea do primeiro domicílio sendo apenas um indivíduo entrevistado. A fim de proporcionar melhor distribuição para efeito de vizinhança, foi sorteado um domicílio e saltado três, iniciando-se sempre pelo lado direito da rua. Não coincidindo a casa escolhida com a presença do adulto, esta resultou em procura no domicílio seguinte, reiniciando-se o processo em cada entrevista. $\mathrm{Na}$ existência de mais de um indivíduo no domicílio, um sorteio simples era realizado para a escolha do entrevistado, desde que contemplasse ao critério de inclusão, que foi ter mais de 18 anos. Foram excluídos aqueles que se recusaram a participar do estudo.

As visitas ao domicílio incluíram a aplicação de um questionário face a face, proposto pelo Ministério da Saúde ${ }^{(2)}$. As variáveis abordadas no presente estudo incluíram: características sociodemográficas como idade; cor (branca, negra, parda ou morena, amarela); situação conjugal (solteiro(a), casado(a)/unido(a), viúvo(a), separado(a)); nível de escolaridade (nunca estudou, ensino fundamental, ensino médio, ensino superior); além das principais características associadas à ocorrência de DCNT como: 1) tabagismo (fumante ativo, independente do número de cigarros, não fumante e ex-fumante); 2) atividade física (considerando ativos no lazer: atividade de intensidade leve ou moderada (caminhada, caminhada em esteira, musculação, hidroginástica, ginástica em geral, natação, artes marciais e luta, bicicleta, voleibol ou outra) por pelo menos 30 minutos diários em 5 ou mais dias da semana ou atividades de intensidade vigorosa (corrida, corrida em esteira, ginástica aeróbica, futebol, basquetebol ou tênis) por pelo menos 20 minutos diários em 3 ou mais dias da semana; ativos na ocupação - realiza esforços físicos intensos no trabalho; ativos no transporte/deslocamento - deslocou para o trabalho caminhando ou de bicicleta; e avaliado como inativo aquele sem atividade física regular, ou seja, com frequência menor que três vezes por semana e duração não inferior a 30 minutos, independente da modalidade do exercício realizado); 3 ) ingestão de bebida alcoólica (consumo de forma abusiva - mais do que cinco doses (homem) ou quatro doses (mulher) em uma única ocasião, pelo menos uma vez nos últimos 30 dias (uma dose de bebida alcoólica corresponde a uma lata de cerveja, uma taça de vinho ou uma dose de qualquer bebida destilada); 4) estado nutricional(analisado pelo Índice de Massa Corporal (IMC) calculado pela formula: peso(kg)/estatura(m) ${ }^{2}$, e classificação em excesso de peso (quando IMC $\geq 25 \mathrm{~kg} / \mathrm{m}^{2}$ ) e obesidade (IMC $\geq 30 \mathrm{~kg} / \mathrm{m}^{2}$ ) no qual peso e altura foram autorreferidos ${ }^{(2)}$.

Para a coleta de dados, utilizou-se um questionário semiestruturado impresso pelos pesquisadores (totalizando $05)$, os quais perguntavam e anotavam as respostas. Não houve tempo pré-estabelecido para o preenchimento completo do instrumento. O próprio entrevistado escolhia o local do domicílio para a realização da entrevista. Após a explanação dos objetivos propostos com a pesquisa e a leitura e assinatura do Termo de Consentimento Livre e Esclarecido (TCLE), o participante era convidado a participar do estudo. Em caso de recusa, um novo indivíduo era questionado e ou outro domicílio selecionado. Um estudo piloto foi realizado para o treinamento prévio das pesquisadoras.

Para a análise dos dados, utilizou-se o software SPSS versão 20.0. Para estudar a associação entre as variáveis independentes e a ocorrência ou não do desfecho, foi realizado análise bivariada das variáveis em cada nível de determinação utilizando os testes Qui-quadrado de 
Pearson, quando as variáveis apresentavam distribuição normal e Exato de Fisher para os dados não paramétricos. Para todos os testes estatísticos inferenciais o nível de significância equivaleu ao valor de $\mathrm{p}<0,05$. A qualidade do ajuste consistiu na aplicação do teste de Hosmer-Lemeshow.

O estudo recebeu aprovação do Comitê de Ética em Pesquisa com Seres Humanos da Universidade Estadual de Maringá (Parecer n³0564/2012).

\section{RESULTADOS}

Participaram do estudo 453 adultos, sendo 77,5\% $(\mathrm{n}=351)$ do sexo feminino. A média de idade foi de $52,0 \pm$ 16,23 anos. A distribuição percentual da população adulta segundo características sociodemográficas se encontra na Tabela I. Ainda, 49,7\% $(\mathrm{n}=225)$ apresentaram baixa escolaridade.

Em relação aos fatores de risco à ocorrência de DCNT, observou-se que $74,2 \%(n=336)$ não realizavam atividade física e $56,6 \%(\mathrm{n}=209)$ apresentavam excesso de peso, dos quais $35,4 \%(n=160)$ estavam com obesidade (Tabela II).
O consumo abusivo de bebida alcoólica não teve proporção elevada na população estudada e houve uma redução no número de fumantes para ex-fumantes em 9,3\% $(\mathrm{n}=44)$.

Na Tabela III, estão os resultados das análises bivariadas entre as variáveis estudadas e os principais fatores de risco para DCNT. Destacou-se significância estatística na prevalência da inatividade física nos indivíduos do sexo feminino, com baixa escolaridade e entre os viúvos (as).

Quanto ao consumo abusivo de álcool, os indivíduos do sexo masculino, com baixa escolaridade e entre os solteiros (as) tiveram significância estatística. O cigarro foi prevalente entre os homens e os solteiros (as) e o excesso de peso entre os indivíduos do sexo masculino, baixa escolaridade e casados(as), com significância estatística para essas variáveis.

Não se observou significância estatística quando os fatores de risco evidenciados foram associados com a característica raça/cor (valor de $\mathrm{p}>0,05$ ).

Tabela I - Distribuição percentual da população adulta segundo características sociodemográficas. Maringá, PR, 2013 $(n=453)$.

\begin{tabular}{lcc}
\hline Variáveis & n & \% \\
\hline Sexo & 102 & 22,5 \\
Masculino & 351 & 77,5 \\
Feminino & & \\
Escolaridade & 21 & 4,6 \\
Nunca estudou & 225 & 49,7 \\
Ensino fundamental & 123 & 27,2 \\
Ensino médio & 84 & 18,5 \\
Ensino superior & & \\
Situação conjugal & 69 & 15,2 \\
Solteiro(a) & 296 & 65,3 \\
Casado(a)/unido(a) & 57 & 12,6 \\
Viúvo(a) & 31 & 6,8 \\
Separado(a)/divorciado(a) & & \\
Cor & 316 & 66,5 \\
Branca & 16 & 3,5 \\
Preta & 117 & 25,8 \\
Parda & 4 & 4,2 \\
Amarela & & \\
\hline
\end{tabular}


Tabela II - Prevalência dos fatores de risco à ocorrência de Doenças Crônicas Não Transmissíveis (DCNTs). Maringá, PR, 2013.

\begin{tabular}{lcc}
\hline Variáveis & $\mathbf{n}$ & $\mathbf{\%}$ \\
\hline Atividade física & & \\
$\quad$ Ativo & 117 & 25,8 \\
$\quad$ Inativo & 336 & 74,2 \\
Bebida alcoólica & & \\
$\quad$ Consumo abusivo & 53 & 11,7 \\
$\quad$ Não abusivo & 400 & 88,3 \\
Tabagismo & 45 & 9,9 \\
$\quad$ Fumantes & 87 & 19,2 \\
Ex-fumantes & 321 & 70,9 \\
$\quad$ Não fumante & & \\
Estado nutricional* & 160 & 43,4 \\
Adequado & 209 & 56,6 \\
$\quad$ Excesso de peso & & \\
\hline
\end{tabular}

$* \mathrm{n}=369$ adultos avaliados

Tabela III - Frequência de fatores de risco para Doenças Crônicas Não Transmissíveis (DCNTs) na população adulta segundo variáveis sociodemográficas. Maringá, PR, 2013.

\begin{tabular}{|c|c|c|c|c|c|c|c|c|}
\hline \multirow[t]{2}{*}{ Variáveis } & \multicolumn{2}{|c|}{$\begin{array}{l}\text { Atividade física } \\
\text { Inativo }\end{array}$} & \multicolumn{2}{|c|}{$\begin{array}{l}\text { Consumo abusivo de } \\
\text { bebida alcoólica }\end{array}$} & \multicolumn{2}{|c|}{ Fumantes } & \multicolumn{2}{|c|}{ Excesso de peso } \\
\hline & $\%$ & p-valor & $\%$ & p-valor & $\%$ & p-valor & $\%$ & p-valor \\
\hline Sexo & & 0,04 & & $<0,01$ & & $<0,01$ & & $<0,01$ \\
\hline Masculino & 66,7 & & 22,5 & & 15,7 & & 58,8 & \\
\hline Feminino & 76,4 & & 7,7 & & 8,3 & & 42,5 & \\
\hline Escolaridade & & $<0,01$ & & $<0,01$ & & 0,79 & & $<0,01$ \\
\hline Nunca estudou & 90,5 & & - & & 14,3 & & 28,6 & \\
\hline Ensino fundamental & 77,8 & & 8,4 & & 9,8 & & 49,8 & \\
\hline Ensino médio & 73,2 & & 11,4 & & 11,4 & & 44,7 & \\
\hline Ensino superior & 61,9 & & 20,2 & & 7,1 & & 42,9 & \\
\hline Situação conjugal & & $<0,04$ & & $<0,03$ & & $<0,01$ & & $<0,04$ \\
\hline Solteiro(a) & 63,8 & & 20,3 & & 17,4 & & 43,5 & \\
\hline Casado(a)/unido(a) & 74,3 & & 10,5 & & 7,8 & & 48,0 & \\
\hline Viúvo(a) & 86,0 & & 5,3 & & 7,0 & & 40,4 & \\
\hline Separado(a)/divorciado(a) & 74,2 & & 6,5 & & 19,4 & & 45,2 & \\
\hline Cor & & 0,86 & & 0,76 & & 0,40 & & 0,06 \\
\hline Branca & 75,3 & & 11,3 & & 9,0 & & 48,7 & \\
\hline Preta & 68,8 & & 18,8 & & 6,3 & & 37,5 & \\
\hline Parda & 72,6 & & 10,3 & & 12,8 & & 42,7 & \\
\hline Amarela & 68,4 & & 5,3 & & 10,5 & & 31,6 & \\
\hline
\end{tabular}

\section{DISCUSSÃO}

As características sociodemográficas mostraram associação entre os principais fatores de risco para as DCNT corroborando com a hipótese inicial do presente estudo, de que tais doenças também podem ter sua origem nos determinantes sociais. Os resultados apontaram relevância estatística entre os fatores de risco como inatividade física, consumo abusivo de bebida alcoólica, tabagismo e excesso de peso em associação com as características sociodemográficas, idade, sexo e escolaridade à semelhança de outros estudos ${ }^{(8,10,11)}$. Esta situação pode estar relacionada às condições de vida de um novo perfil da população que está mais independente e exposta a práticas de comportamentos 
sedentários e hábitos de vida nocivos para a saúde. Esse conjunto de fatores de riscos modificáveis responde pela grande maioria das mortes e por frações importantes da carga de doenças relacionadas às $\mathrm{DCNT}^{(8)}$.

Quanto a não realização de atividade física regular no presente estudo, foi possível observar associação com significância estatística entre os indivíduos do sexo feminino, baixa escolaridade e viúvos (as). O índice encontrado $(76,4 \%)$ foi semelhante ao encontrado em Pelotas, RS, onde a inatividade física foi o fator de risco mais prevalente encontrado $(75,6 \%)$ sendo mais comum entre as mulheres ${ }^{(8)}$. Destaca-se que a baixa escolaridade pode ter contribuído para maiores proporções encontradas na presente pesquisa, uma vez que a ausência de práticas de atividade física permaneceu entre aquelas que nunca haviam estudado $(90,5 \%)$.

Outro estudo epidemiológico com adultos fisicamente inativos apresentou em suas análises que o comportamento sedentário, em especial a quantidade de tempo gasto assistindo televisão, foi mais prevalente entre as mulheres $^{(11)}$. Há uma tendência crescente mundial de que as pessoas se tornem cada vez menos ativas em razões dos avanços tecnológicos e atividades lúdicas passivas ${ }^{(15)}$. Entre as mulheres, esses achados podem estar relacionados à inserção desse público no mercado de trabalho e ou a sua não inserção, estando reservadas apenas para as atividades domésticas $^{(16)}$.

São necessárias políticas e ações que incentivem a realização de exercícios regulares evidenciando seus benefícios e garantindo subsídios para a estruturação de locais destinados a este fim $^{(8,15)}$. Além disso, estima-se que 2,1 milhões de dólares por ano poderiam ser economizados nos cuidados em saúde por meio da prática regular de exercícios físicos ${ }^{(17)}$. A prescrição e a prática de exercício regular é eficaz na melhora do quadro clínico de indivíduos como na redução da pressão sanguínea e hemoglobina glicosilada, resultando na prevenção e controle de doenças hipertensivas e dos distúrbios do metabolismo da glicemia, bem como possui efeitos positivos importantes sobre a saúde mental, com melhora na função cognitiva e redução de depressão, demência e doença de Alzheimer ${ }^{(18)}$.

Vale ressaltar que a não realização de atividade física, além de outros fatores, como tabagismo, padrão de sono alterado, uso abusivo de álcool também contribuem para o surgimento do excesso de peso resultando no agravamento de doenças crônicas e dislipidemias ${ }^{(10,16)}$.

Assim como em outros estudos, o excesso de peso foi o segundo fator de risco prevalente evidenciado na presente pesquisa, com significância estatística para os indivíduos do sexo masculino, baixa escolaridade e os $\operatorname{casados}^{(10,18)}$. No Brasil, o aumento do excesso de peso é cada vez mais frequente entre os homens $(54,7 \%)$ em relação às mulheres $(47,4 \%)$, e naqueles com menos de oito anos de estudo $(58,1 \%)^{(19)}$. Destaca-se que a obesidade aumenta em 2,9 vezes o risco de desenvolvimento de hipertensão arterial e diabetes mellitus ${ }^{(15)}$.

Evidências mostram que tanto para homens quanto para as mulheres, os principais riscos de saúde relacionados ao estilo de vida como fumo, álcool, dieta pouco saudável, obesidade e sedentarismo são responsáveis por mais de três milhões de mortes por ano no mundo, com riscos relativos semelhantes em homens e mulheres para sobrepeso e obesidade. Fumo e álcool são as principais causas das disparidades globais de gênero na mortalidade ${ }^{(20)}$.

Outro ponto relevante apontado pelo presente estudo revelou que o consumo abusivo de bebida alcoólica foi mais frequente entre os homens solteiros e com escolaridade superior a 12 anos de estudo, demonstrando índices semelhantes ao encontrado em outros estados e nas demais capitais brasileiras ${ }^{(21,22)}$. O consumo de álcool corresponde a 2,3 milhões de mortes no ano, além de projetar um impacto negativo nos cenários da saúde, social, políticos e econômicos ${ }^{(23)}$. Há uma tendência mundial de este consumo ser mais frequente entre jovens tendendo a aumentar com o nível de escolaridade ${ }^{(21)}$.

Assim como o consumo do álcool, o tabagismo também foi evidente no universo pesquisado no presente estudo com destaque para os homens de baixa escolaridade e entre os separados/viúvos, assim como outros estudos realizados nas capitais brasileiras ${ }^{(21,24)}$. A literatura evidencia que o tabagismo é responsável por aproximadamente 5,1 milhões de mortes por ano no mundo e que o ato de fumar seja a causa de $71 \%$ dos cânceres de pulmão ${ }^{(25)}$.

A presente pesquisa pode identificar a prevalência dos fatores de risco à ocorrência das DCNT no nível local, o que pode fornecer subsídios para o planejamento, implementação e avaliação de intervenções em saúde pública voltadas para a redução destas doenças. Não foram explorados outros fatores cuja presença poderia afetar a probabilidade de acúmulo de fatores de risco para DCNT, como a utilização de serviços de saúde por exemplo. A interpretação dos resultados da pesquisa deve ser feita à luz de algumas limitações metodológicas. Sistemas de monitoramento que se baseiam em dados referidos apresentam certas limitações que devem ser consideradas na interpretação dos dados. A acurácia das informações autorreferida depende do conhecimento dos respondentes de informações relevantes, da capacidade de recordá-las e da fidedignidade ao recordálas. Portanto, a situação real da frequência dos fatores de risco para as DCNT possivelmente pode ser pior do que a encontrada, configurando uma limitação do estudo. Nessa perspectiva, os principais fatores de risco evidenciados 
a partir do desenvolvido da atual pesquisa poderiam ser evitáveis, a partir da introdução de medidas sociais, de prevenção e controle da saúde, como a prática regular de atividade física, alimentação equilibrada e a adoção de hábitos saudáveis. Ademais, monitorar os fatores de risco mais prevalentes na população pode subsidiar e permear a introdução de medidas diferenciadas para a prática clínica por profissionais da área da saúde, ao coordenar a gestão do cuidado da população atendida visando ao desencadeamento de intervenções e ações articuladas com um plano terapêutico baseado em evidências e centrado nas reais necessidades dessa população.

Dessa maneira, sugere-se que novas pesquisas sejam elencadas nesse âmbito.

\section{CONCLUSÃO}

Neste estudo, grande parte dos fatores de risco, identificados à ocorrência das doenças crônicas não transmissíveis como inatividade física, consumo abusivo de bebida alcóolica, tabagismo e excesso de peso, é passível de modificações a partir da incorporação de hábitos saudáveis e mudanças no comportamento e estilo de vida. A prevalência desses fatores neste estudo esteve associada estatisticamente ao sexo, à idade, à escolaridade e à situação conjugal.

A inatividade física foi o fator de risco mais prevalente e esteve associada aos indivíduos do sexo feminino, bem como baixa escolaridade e vivos (as). Por outro lado, o gênero masculino esteve associado ao consumo abusivo de bebida alcoólica, do tabagismo e do excesso de peso.

\section{REFERÊNCIAS}

1. Duarte EC, Barreto SM. Transição demográfica e epidemiológica: a epidemiologia e serviços de saúde revista atualiza o tema. Epidemiol Serv Saúde. 2012;21(4):529-32.

2. Ministério da Saúde (BR), Secretaria de Vigilância em Saúde.PlanodeAções Estratégicas para oEnfrentamento das Doenças Crônicas Não Transmissíveis (DCNT) no Brasil 2011-2022 [Internet]. Brasília: Ministério da Saúde; 2011 [acesso em 2015 Jul 7]. Disponível em: http://www.sbn.org.br/noticias/acoes_estrategicas.pdf

3. Malta DC, Iser BPM, Claro RM, Moura L, Bernal RTI, Nascimento AF, et al. Prevalência de fatores de risco e proteção para doenças crônicas não transmissíveis em adultos: estudo transversal, Brasil, 2011. Epidemiol Serv Saúde. 2013;22(3):423-34.

4. Malta DC, Gosch CS, Buss P, Rocha DG, Rezende R, Freitas PC, et al. Doenças Crônicas Não Transmissíveis e o suporte das ações intersetoriais no seu enfrentamento. Ciênc Saúde Coletiva. 2014;19(11):4341-50.

5. Paulo TRS, Gomes IC, Santos VR, Christofaro DGD, Castellano SM, Freitas IF Júnior. Atividade física e estado nutricional: fator de proteção para doenças crônicas não transmissíveis (DCNT) em idosas. Rev Bras Promoç Saúde. 2014;27(4):527-32.

6. Schmidt MI, Duncan BB, Silva GA, Menezes AM, Monteiro CA, Barreto SM, et al. Doenças crônicas não transmissíveis no Brasil: carga e desafios atuais. Lancet [Internet]. 2011 [acesso em $2015 \mathrm{Jul}$ 7];(4):61-74. Disponível em: http://dms.ufpel.edu. br/ares/bitstream/handle/123456789/222/1\%20\%20 2011\%20Doen $\%$ C3\%A7as\%20cr\%C3\%B4nicas\%20 n\%C3\%A3o\%20transmiss\%C3\%ADveis\%20no\%20 Brasil.pdf? sequence $=1$

7. Silva LS, Cotta RMM, Rosa COB. Estratégias de promoção da saúde e prevenção primária para enfrentamento das doenças crônicas: revisão sistemática. Rev Panam Salud Publica. 2013;34(5):34350 .

8. Muniz LC, Schneider BC, Silva ICM, Matijasevich A, Santos IS. Fatores de riscos comportamentais acumulados para doenças cardiovasculares no sul do Brasil. Rev Saúde Pública. 2012;46(3):534-42.

9. Malta DC, Silva SA, Oliveira PPV, Iser BPM, Bernal RTI, Sardinha LMV, et al. Resultados do monitoramento dos Fatores de risco e Proteção para Doenças Crônicas Não Transmissíveis nas capitais brasileiras por inquérito telefônico, 2008. Rev Bras Epidemiol. 2012;15(3):639-50.

10. Liu Y, Croft JB, Wheaton AG, Kanny D, Cunningham $\mathrm{TJ}, \mathrm{LuH}$, et al. Clustering of five health-related behaviors for chronic disease prevention among adults, United States, 2013. Prev Chronic Dis. 2016;13:(1):600-54.

11. Healy GN, Dunstan DW, Salmon J, Shaw JE, Zimmet PZ, Owen N. Television time and continuous metabolic risk in physically active adults. MedSci Sports Exerc. 2008;40(4):639-45.

12. Pitilin EB, Gutubir D, Molena-Fernandes CA, Pelloso SM. Internações sensíveis à atenção primária específicas de mulheres. Ciênc Saúde Coletiva. 2015;20(2):44148.

13. Instituto Paranaense de Desenvolvimento Econômico e Social. Caderno Estatístico: Município de Maringá. Maringá: Instituto Paranaense de Desenvolvimento Econômico e Social; 2015 [acesso em 2015 Jul 5]. Disponível em: http://www.ipardes.gov.br/cadernos/ MontaCadPdf1.php?Municipio $=87000$ 
14. Instituto Brasileiro de Geografia e Estatística - IBGE. Ministério do Planejamento, orçamento e gestão. Primeiros resultados do Censo 2010. Brasília: IBGE; 2011.

15. Ministério da Saúde (BR), Agência Nacional de Saúde Suplementar. Manual técnico para Promoção da saúde e prevenção de riscos e doenças na saúde suplementar. $4^{\mathrm{a}}$ ed. Rio de Janeiro: Agência Nacional de Saúde Suplementar; 2011 [acesso em 2015 Jul 10]. Disponível em: http://www.ans.gov.br/images/stories/ Materiais_para_pesquisa/Materiais_por_assunto/ manual_promoprev_web.pdf

16. Adserà A, Ferrer A. Occupational skills and labour market progression of married immigrant women in Canada. Labour Econ [Internet]. 2016 [acesso em 2016 Jun 27];39:88-98. Disponível em: http://www.ncbi. nlm.nih.gov/pubmed?Db=pubmed $\& \mathrm{Cmd}=$ Retrieve $\&$ li st_uids $=27217617 \&$ dopt $=$ abstractplus

17. Jen Kruger J, Brennan A, Strong M, Thomas C, Norman $\mathrm{P}$, Epton T. The cost-effectiveness of a theory-based online health behaviour intervention for new university students: an economic evaluation. BMC Public Health. 2014;1(4):1014-21.

18. Thornton JS, Frémont P, Khan K, Poirier P, Fowles J, Wells GD, et al. Physical activity prescription: a critical opportunity to address a modifiable risk factor for the prevention and management of chronic disease: a position statement by the Canadian Academy of Sport and Exercise Medicine. Br J Sports Med [Internet]. 2016 [acesso em 2016 Jun 27]:1-6. Disponível em: http://bjsm.bmj.com/content/early/2016/06/22/ bjsports-2016-096291.full.pdf + html

19. Ministério da Saúde (BR), Secretaria de vigilância em saúde. Vigilância de fatores de risco e proteção para doenças crônicas por inquérito telefônico: estimativas sobre a frequência e distribuição sócio demográfica de fatores de risco e proteção para doenças crônicas nas capitais dos 26 estados brasileiros e no Distrito Federal em 2013. Brasília: Ministério da Saúde; 2014 [acesso em 2015 Jul 10]. Disponível em: https://biavati.files. wordpress.com/2014/05/vigitel-2013.pdf
20. Eshre C. The influence of social factors on gender health. Hum Reprod. 2016;15(4):1110-20.

21. Ministério da Saúde (BR), Secretaria de Vigilância em Saúde, Secretaria de Gestão Estratégica e Participativa. Vigitel Brasil 2011: vigilância de fatores de risco e proteção para doenças crônicas por inquérito telefônico. Brasília: Ministério da Saúde; 2012 [acesso em 2015 Jul 11]. Disponível em: http://www.abeso.org. br/uploads/downloads/75/553a276c33350.pdf

22. Martins LN, Souza LS, Silva CF, Machado RS, Silva CEF, Vilagra MM, et al. Prevalência dos fatores de risco cardiovascular em adultos admitidos na unidade de dor torácica de Vassouras, RJ. Rev Bras Cardiol. 2011;24(5):299-307.

23. Nunes JM, Campolina LR, Vieira MA, Caldeira AP. Consumo de bebidas alcoólicas e prática do bingedrinking entre acadêmicos da área da saúde. Rev Psiquiatr Clín. 2012;39(3):94-9.

24. Malta DC, Oliveira TP, Luz M, Stopa SR, Silva JB Junior, Reis AAC. Tendências de indicadores de tabagismo nas capitais brasileiras, 2006 a 2013. Ciênc Saúde Coletiva. 2014;20(3):631-40.

25. Carmo CBS, Silva RD, Teixeira RC. Perfil epidemiológico de pacientes com câncer de pulmão em hospital público de referência oncológica do estado do Pará. Rev Para Med. 2014;28(1):55-62.

\section{Endereço do primeiro autor:}

Érica de Brito Pitilin

Universidade Federal da Fronteira Sul - UFFS

Avenida Nereu Ramos, 519E

Bairro: Centro

CEP: 89.802-020 - Chapecó - SC - Brasil

E-mail: erica.pitilin@gmail.com

\section{Endereço para correspondência:}

Daiane Schuck

Universidade Federal da Fronteira Sul - UFFS

Avenida Nereu Ramos, 519E

Bairro: Centro

CEP: 89.802-020 - Chapecó - SC - Brasil

E-mail: daya_schuck@hotmail.com 